\title{
Performance of Temporal Artery Temperature Measurement in Ruling Out Fever: Implications for COVID-19 Screening
}

J Gen Intern Med 35(11):3398-400

DOI: $10.1007 / \mathrm{s} 11606-020-06205-2$

(c) Society of General Internal Medicine 2020

\section{INTRODUCTION}

Current CDC recommendations for mitigation of community COVID-19 transmission include temperature screening. ${ }^{1}$ Due to low cost and ease-of-use, temporal artery thermometers (TATs) applied to the forehead are widely employed to screen for fever, but prior literature has suggested poor sensitivity and high variability. ${ }^{2,3}$ Published data are largely limited by small per-study sample sizes and a focus on pediatric, surgical, and intensive care settings that are not generalizable to real-world screening populations.

We sought to determine the real-world test performance of TATs for fever rule-out by utilizing a large electronic dataset of emergency department encounters for whom universal temperature screening was conducted. For reference standards, we included rectal temperature, a widely recognized core temperature, as well as oral temperature, which benefits from wide clinical acceptance and robust specificity. ${ }^{2}$ Our primary objective was to determine TAT sensitivities and specificities across a range of temperatures in comparison with rectal and oral cutoffs of $100.4{ }^{\circ} \mathrm{F} / 38{ }^{\circ} \mathrm{C}$. Our secondary outcome was limit-ofagreement (LOA) by Bland-Altman analysis.

\section{METHODS}

We extracted temperature measurements and method of temperature assessment from electronic health record (EHR) data (Epic, Verona, WI) collected between March 2013 and June 2019 within a large hospital system comprising ten acute care sites. We identified paired perpatient data where a TAT measurement was documented within 15 minutes of a rectal temperature measurement or oral temperature. When multiple measurements were taken with a single modality within the defined interval, the mean value was used. In the Bland-Altman analysis, we

Received June 19, 2020

Accepted August 31, 2020

Published online September 15, 2020 calculate the mean of the differences between the paired measurements and the limits-of-agreement (LOA) as defined by $95 \%$ confidence interval bounds.

\section{RESULTS}

We identified 1.84 million adult (age $>18$ years) emergency department visits by 602,089 patients with over 4.6 million temperature readings; there were 1293 paired readings from 1276 encounters that met our inclusion for TAT versus rectal measurement and 16,132 readings from 16,031 encounters for TAT versus oral measurement. The admission rate across the paired measurement patient population was $50.0 \%$. The prevalence of fever in the rectal and oral temperature populations was $34.4 \%$ and $4.3 \%$, respectively. Using a threshold of $100.4{ }^{\circ} \mathrm{F}$, TAT measurement identified fever compared with the rectal reference with sensitivity 0.27 (95\% CI $0.27-0.31$ ), specificity 0.98 (0.96-0.99), PPV 0.85 (0.79-0.91), and NPV $0.72(0.69-0.74)$. TAT measurement identified fever compared with the oral reference with a sensitivity 0.23 (95\% CI $0.20-0.26)$, specificity $0.99(0.99-0.99)$, PPV $0.53(0.48-$ $0.59)$, and NPV 0.97 (0.96-0.97). We did not observe significant differences in fever sensitivity when limiting our paired analysis to 5- or 10-minute windows. Decreasing the threshold for fever using TAT improved sensitivity with respect to the rectal temperature gold standard of $100.4^{\circ} \mathrm{F}$ (Fig. 1). Using a TAT cutoff of $99^{\circ} \mathrm{F}$, for example, increased sensitivity to 0.63 $(0.58-0.67)$ and NPV to $0.82(0.79-0.84)$, while specificity decreased to $0.86(0.83-0.88)$ and PPV to $0.7(0.65-0.74)$. The mean differences and LOA between TAT and rectal temperatures and TAT and oral temperatures were -1.13 $(\mathrm{LOA}-6.69 \text { to } 4.42)^{\circ} \mathrm{F}$, and $-0.45(-3.89-2.99)^{\circ} \mathrm{F}$, respectively (Fig. 2).

\section{DISCUSSION}

Consistent with prior work, we found TATs had poor test performance, identifying less than one in three positive cases. ${ }^{2-4}$ We observed that decreasing TAT temperature thresholds yielded significantly improved test sensitivity with modest losses in specificity and note that this approach is being recommended by some state departments of public health. ${ }^{5}$ Generalizability of our analysis may be 


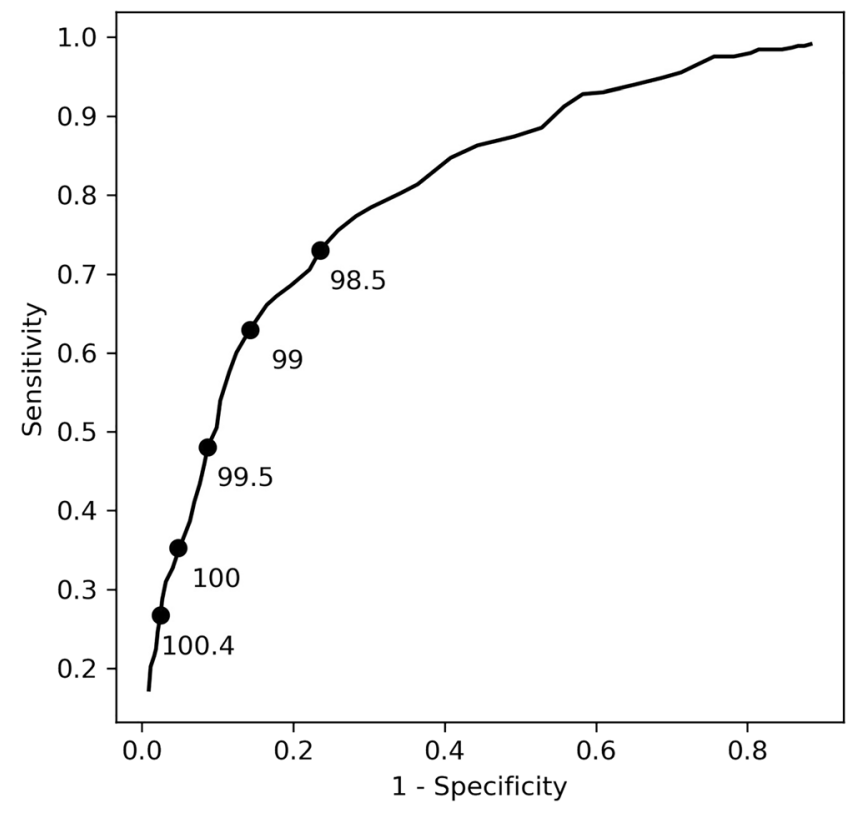

Figure 1 Receiver operating characteristic plot for fever screening by TAT compared with rectal fever of $100.4^{\circ} \mathrm{F}$. Select temperature cutoffs for TAT are labeled.

limited by reliance on emergency department visits with paired measures, as well as possible non-uniformity of testing devices over time. ${ }^{4}$

Based on current $\mathrm{CDC}$ recommendations for fever measurement (cutoff of $100.4^{\circ} \mathrm{F}$ ) and TAT screening, the majority of people who would meet the criteria for fever would be wrongly classified as afebrile. A lower TAT fever cutoff would necessarily increase false positive rates, likely prompting testing for COVID-19 and potentially unnecessary self-isolation and workplace withdrawal for uninfected individuals. Public and private entities seeking to mitigate the transmission of COVID-19 in the workplace or high-risk settings should consider a combination of lower thresholds (e.g., $99^{\circ} \mathrm{F}$ ), alternative technologies, or serial temperature assessment approaches as well as using temperature alongside symptom screening to ensure that public health goals are achieved. ${ }^{6}$
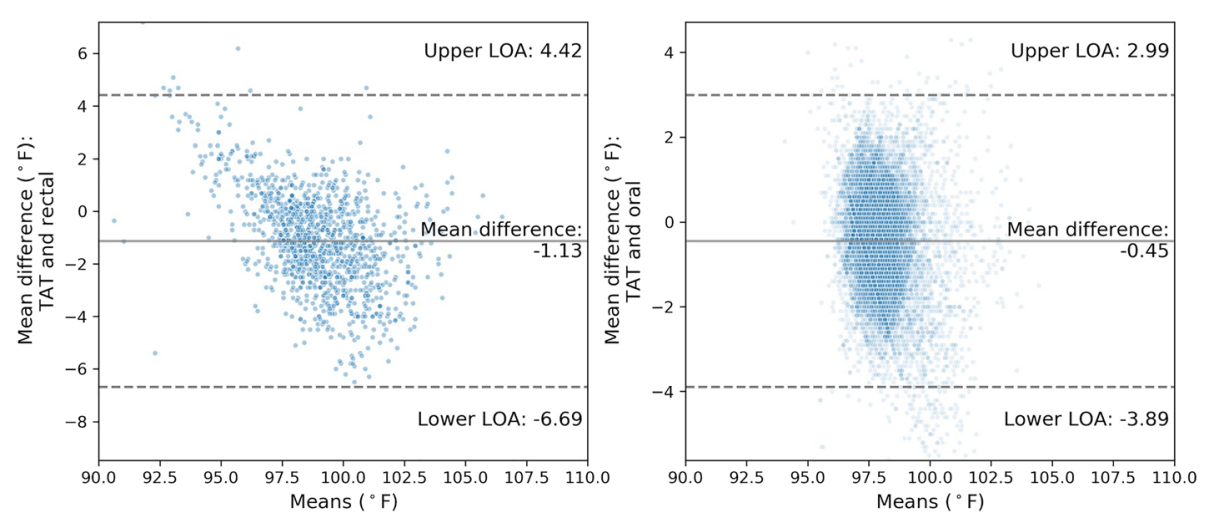

Figure 2 Bland-Altman plots of TAT and a rectal and b oral measurements. Mean difference is shown with the solid line, limits of agreement as defined by two standard deviations are shown with dashed lines.
Acknowledgments: IRB: This work was deemed exempt by the Yale Institutional Review Board.

Adrian D. Haimovich, $M D, P h D^{1}$

R. Andrew Taylor, MD, MHS ${ }^{1}$

Arjun K. Venkatesh, MD, MBA, MHS ${ }^{1,3}$

${ }^{1}$ Yale Department of Emergency Medicine, Yale School of Medicine,

New Haven, CT, USA

${ }^{2}$ Yale Department of Internal Medicine, Section of Cardiovascular Medicine, Yale School of Medicine,

New Haven, CT, USA

${ }^{3}$ Yale New Haven Hospital Center for Outcomes

Research and Evaluation,

New Haven, CT, USA

Corresponding Author: Adrian D. Haimovich, $M D, P h D$; Yale Department of Emergency Medicine, Yale School of Medicine, New Haven, CT, USA (e-mail: adrian.haimovich@yale.edu).

Authors' Contribution Author tasks: All authors conceived of this project and were involved in manuscript preparation. ADH and RAT performed the data processing and analysis.

\section{Compliance with Ethical Standards:}

Conflict of Interest: The authors report no conflict of interests nor specific funding for this work.

\section{REFERENCES}

1. Centers for Disease Control and Prevention. Implementation of Mitigation Strategies for Communities with Local COVID-19 Transmission. Published online March 10, 2020:10.

2. Niven DJ, Gaudet JE, Laupland KB, Mrklas KJ, Roberts DJ, Stelfox HT. Accuracy of Peripheral Thermometers for Estimating Temperature: A Systematic Review and Meta-analysis. Ann Intern Med. 2015;163(10):768. https://doi.org/10.7326/M15-1150.

3. Geijer H, Udumyan R, Lohse G, Nilsagård Y. Temperature measurements with a temporal scanner: systematic review and meta-analysis. BMJ Open. 2016;6(3):e009509. https://doi.org/10.1136/bmjopen-2015-009509.

4. Bijur PE, Shah PD, Esses D. Temperature measurement in the adult emergency department: oral, tympanic membrane and temporal artery temperatures versus rectal temperature. Emerg Med J. 2016;33(12):843847. https://doi.org/10.1136/emermed-2015-205122.

5. Delaware Health and Social Services Division of Public Health. Essential Services Screening Recommendations for COVID-19 Pandemic. Accessed
Harlan M. Krumholz, $M D, S^{2,3}$ 
June 3, 2020. https://coronavirus.delaware.gov/wp-content/uploads/ sites/177/2020/05/5.7-Essential-Services-Screening-Policy.pdf.

6. Chow EJ, Schwartz NG, Tobolowsky FA, et al. Symptom Screening at Illness Onset of Health Care Personnel With SARS-CoV-2 Infection in King County, Washington. JAMA. 2020;323(20):2087. https://doi.org/10. 1001/jama.2020.6637.
Publisher's Note: Springer Nature remains neutral with regard to jurisdictional claims in published maps and institutional affiliations. 